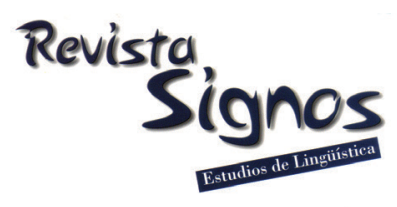

\title{
La contribución del oyente a la interacción oral en Español como Lengua Extranjera. Un estudio comparativo ${ }^{1}$
}

\author{
The contribution of the listener to verbal interaction in \\ Spanish Foreign Language. A comparative study \\ Javier Pérez Ruiz \\ Wenzao Ursuline College of Languages \\ TAIWÁN \\ javiertaiwan@yahoo.es
}

Recibido: 08-IV-2013 / Aceptado: 16-XII-2013

\section{Resumen}

El conocimiento de los factores que pueden impulsar la adquisición de la competencia comunicativa en una segunda lengua es un tema de gran relevancia investigadora por las consecuencias teóricas y didácticas que conlleva. Desafortunadamente, el papel del oyente en la comunicación oral en Español como Lengua Extranjera (ELE) ha sido uno de los factores menos estudiado. Ante esta situación, el propósito fundamental de este artículo es analizar las aportaciones que el oyente realiza en el desarrollo de la competencia comunicativa en ELE. Dos objetivos específicos conducen la investigación: por un lado, se describe la contribución de las señales verbales del oyente a la interacción oral en ELE. Por otro lado, se explora el impacto que el nivel lingüístico en la lengua meta ejerce sobre la producción de los apoyos verbales del oyente. Para lograr estos objetivos, desde el marco teórico del Análisis de la Conversación, se ha seguido una metodología inductiva basada en la elaboración de un corpus oral de 30 conversaciones diádicas en español, mantenidas por estudiantes taiwaneses de dos niveles lingüísticos diferentes de ELE. Los resultados indican que las señales del oyente en la conversación son un componente esencial que contribuye a mejorar la interacción oral en ELE; además, el nivel lingüístico es un factor determinante en las aportaciones que realiza el oyente. Las conversaciones se hacen más naturales y fluidas gracias a la escucha más activa y participativa de los estudiantes de mayor nivel, quienes desarrollaron estrategias cuantitativa y cualitativamente diferentes a los estudiantes de menor nivel.

Palabras Clave: Análisis de la Conversación, Interacción Oral, Turnos de Apoyo, Español Lengua Extranjera. 


\begin{abstract}
The knowledge of the factors that can promote the acquisition of communicative competence in a second language is an issue of great relevance to researchers due to the theoretical and didactic consequences involved. However, far too little attention has been paid to the role of the listener in oral communication in Spanish foreign language (SFL). So the aim of this study is to analyze the contributions of the listener in the development of communicative competence in SFL. The first specific objective of this study is to describe the participation of the listener responses emitted by the listener in the verbal interaction in SFL. The second is to explore the impact that language proficiency in the target language production, has on listener responses. To achieve these objectives, from the theoretical framework of Conversation Analysis, an inductive methodology based on the elaboration of an oral corpus of 30 dyadic Spanish conversations in Taiwanese SFL learners of two different linguistic levels of SFL, has been adopted. The results indicate that listener responses in conversations are essential components that contribute to improve the oral interaction in SFL, and show that language proficiency is a relevant factor in the contributions made by the listener. Conversations become more fluent and spontaneous through more active and participatory listening from the higher level students, who used quantitatively and qualitatively different listener responses.
\end{abstract}

Key Words: Conversation Analysis, Verbal Interaction, Listener Responses, Spanish Foreign Language.

\title{
INTRODUCCIÓN
}

El Marco Común Europeo de Referencia para las Lenguas (MCER) fijó la adquisición de la competencia comunicativa como una de las metas fundamentales en el proceso de aprendizaje de una nueva lengua (Consejo de Europa, 2002). A pesar de estar bien establecidos los fundamentos teóricos de la competencia comunicativa, una de las preocupaciones principales de los profesores de Español como Lengua Extranjera (ELE) sigue siendo cómo promover entre sus estudiantes la interacción oral $(\mathrm{IO})$ en la lengua meta. Ante esta situación, parece recomendable que antes de buscar nuevas metodologías que ayuden a adquirir la competencia comunicativa, sea necesario describir de manera fehaciente aquellos fenómenos lingüísticos y metalingüísticos que puedan impulsar la IO en ELE.

Uno de los factores implicados en la IO menos estudiados ha sido analizar la contribución del oyente al acto comunicativo. Históricamente, el estudio de la IO enfatizó la contribución del hablante y subordinó el papel del oyente a mero espectador, cuyas aportaciones léxicas y metalingüísticas fueron consideradas como fenómenos marginales al acto comunicativo. Las nuevas corrientes sociolingüísticas de la segunda mitad del siglo XX, entre las que destacamos las aportaciones del Análisis de la Conversación (AC), a través de sus estudios empíricos e inductivos 
establecieron un nuevo marco comunicativo, que entendía la conversación como una construcción conjunta, desplegada por ambos interlocutores, hablante y oyente (Vázquez, 2000; Gardner, 2002; Hutchby \& Wooffitt, 2008). El oyente, a través de señales verbales y no verbales, que denominaremos en este artículo-según el término acuñado por Cestero (2000a) - 'turnos de apoyo' (TA), participa de manera activa en la construcción del turno de habla; y su aportación resulta esencial al acto comunicativo (Schegloff, 1982; Ishida, 2006). La monitorización activa y participativa que el oyente elabora durante el turno del hablante goza de una larga tradición investigadora en lengua inglesa, que contrasta con la escasez de estudios realizados en ELE (Berry, 1994; Garcés \& Bou, 2004; Pérez Ruiz, 2011a).

Ante esta situación expuesta, el objetivo general de este artículo es describir el papel del oyente en la adquisición de la competencia comunicativa en ELE. De manera concreta, dos objetivos específicos guían esta investigación. En primer lugar, se examina la contribución de las señales verbales del oyente a la IO en la lengua meta. En segundo lugar, el estudio describe el impacto del nivel lingüístico en ELE sobre la producción de los TA verbales.

Para lograr los mencionados objetivos se ha seguido la metodología propuesta por el AC y la Sociología Variacionista. Se ha realizado un estudio empírico, descriptivo y transversal, basado en un corpus oral representativo de 30 conversaciones diádicas en español, mantenidas por estudiantes taiwaneses de ELE, cuya lengua materna es el chino mandarín. Se describirán de manera cuantitativa y cualitativa las diferentes estrategias comunicativas utilizadas por el oyente en dos diferentes niveles lingüísticos de ELE, nivel B1 y B2 del MCER.

La estructura del artículo describirá en el marco teórico las controversias actuales sobre el tema y definirá, de manera coherente, la terminología y clasificación que se adoptarán. En el apartado de metodología se expondrán los detalles del corpus oral elaborado. A continuación, se mostrarán y discutirán los principales resultados cuantitativos y cualitativos obtenidos. El estudio finalizará enumerando sus principales conclusiones.

Una doble justificación, académica y didáctica, constituyen la originalidad y significación de este artículo. Por un lado, ante la escasez de estudios y las controversias teóricas sobre el tema, se establece una sólida descripción de la producción verbal del oyente en ELE. La investigación confirma el valor esencial de las señales del oyente como un factor fundamental que favorece la adquisición de la competencia comunicativa en ELE. Por otro lado, la evidencia empírica ofrecida en este trabajo está permitiendo diseñar propuestas didácticas, para incorporar en la clase de ELE el aprendizaje explícito de las mencionadas señales verbales del oyente ${ }^{2}$. 


\section{Marco teórico}

\subsection{Competencia comunicativa, Análisis de la Conversación y papel del oyente}

Desde los albores de la civilización occidental se ha postulado el papel esencial del lenguaje en la construcción de la dimensión social del ser humano. Sin embargo, en el ámbito de la lingüística, hubo de esperarse hasta la segunda década del siglo XX para que la mentalidad lingüística dominante, cediera ante una nueva concepción sociolingüística de la lengua (Hutchby \& Wooffit, 2008). Este cambio de mentalidad fue posible gracias a la aportación fundamental de cuatro disciplinas: la Pragmática, la Sociolingüística, el Análisis del Discurso y el Análisis de la Conversación (García, 2009). La culminación y fundamentación teórica de este proceso quedaron reflejadas en la redacción del MCER, que subrayó la significación de la competencia comunicativa en la adquisición de la lengua, y entendía que este concepto aglutinaba elementos lingüísticos, sociolingüísticos y pragmáticos (Consejo de Europa, 2002). Nuestro trabajo investigador, insertado en el espíritu del MCER, adoptará como marco teórico los presupuestos teóricos y metodológicos del AC. Este enfoque, de mentalidad sociolingüística, estudia el habla en la interacción a través de un método inductivo, que analiza muestras de habla en contexto naturales (Moreno, 1998; Hutchby \& Wooffitt, 2008).

Dentro de la competencia comunicativa, la competencia conversacional desempeña un papel crucial, ya que se encuentra en la raíz de la adquisición del lenguaje como primera expresión de la interacción y de la socialización (Levinson, 1983). Para el AC, la conversación no es una actividad desordenada, ni una elaboración cognitiva premeditada del hablante con la exclusiva finalidad de transmitir un contenido referencial a un oyente pasivo. Fundamentalmente, la conversación es un proceso creativo de interacción social, que posee una organización socio-secuencial (Schegloff, 1982; Moreno, 1998; Cestero, 2005). Cestero (2012) definía la conversación como una unidad de interacción social, que se construye por medio de la sucesión ordenada de turnos formulados por los interlocutores de manera alternativa.

Otros autores también han destacado este carácter alternante conversacional (Gallardo Paúls, 1991, 1992; Padilla, 2004). Aunque, de manera general, quien posee el turno de habla se denomina 'hablante', y quien no lo posee se denomina 'oyente'; ambos interlocutores no deben ser definidos como entidades fijas sino cambiantes. Concretamente, Gallardo Paúls (1992) consideraba la conversación como un proceso simultáneo, acumulativo y, sobre todo, dinámico. Los papeles de los interlocutores, hablante y oyente, no pueden ser comprendidos como roles estáticos, sino alternables, es lo que la autora denominaba 'dinamismo conversacional' (Gallardo Paúls, 1991). También Padilla (2004) indicó que durante la conversación el hablante y el oyente intercambiaban sus papeles comunicativos. Este autor sostenía que la distinción entre 
turnos e intervenciones permitía diferenciar dos papeles socio-conversacionales, a saber, el hablante y el oyente (Padilla, 2004). El hablante no solamente es el emisor de contenidos referenciales, además, en cierta medida es oyente porque está atento a las señales verbales y no verbales del oyente. Por su parte, el oyente no se ciñe a la mera labor de escucha pasiva, sino que participa de manera activa por medio de un lenguaje lingüístico y metalingüístico.

En su revisión del tema, Vázquez (2000) recordaba que los primeros estudios de la conversación versaban sobre las contribuciones del hablante, que era el interlocutor activo y la única autoridad, quedando relegado el oyente a un segundo plano. Sin embargo, uno de los logros del AC ha sido considerar que la monitorización activa del oyente resulta fundamental para que progrese la interacción. En el desarrollo de la competencia comunicativa, Padilla (2004) defendía que aprender a conversar es, por un lado, aprender a ser hablante y saber expresarse, pero, además, es aprender a ser oyente, es decir, se desarrolla la competencia comunicativa cuando atendemos a lo que se está diciendo.

Numerosos investigadores han subrayado la aportación esencial que realiza el oyente en la construcción de la conversación (Vázquez, 2000; Gardner, 2001; Garcés \& Bou, 2004; Ishida, 2006). La conversación es una cooperación negociada constituida por elementos lingüísticos (gramaticales y léxicos), por un contexto común que comparten los interlocutores y por la monitorización o feedback que realiza el oyente (Gumperz, 1982). El oyente durante la IO no realiza un acto pasivo, sino que produce una escucha activa y productiva por medio de señales verbales y no verbales, que aunque de escaso valor semántico referencial, desempeñan un papel esencial en las conversaciones porque nos ofrecen datos sobre los principios que rigen la organización de la IO (Gallardo, 1992; Vázquez, 2000; Heinz, 2003; Bangerter \& Clark, 2003). En el pasado, el estudio de estas señales verbales del oyente fueron eliminadas de los corpus orales por ser consideradas marginales. Sin embargo, otro de los méritos del AC ha sido formular, entre sus principios, que en cualquier análisis conversacional nunca deben ser eliminados detalles por ser considerados a priori como irrelevantes. El habla en la interacción abarca mucho más que las palabras grabadas pronunciadas por los interlocutores, ya que las conversaciones naturales comprenden numerosos detalles que deben ser recogidos, transcritos y analizados de manera minuciosa (Seedhouse, 2004; Liddicoat, 2007; Hutchby \& Wooffitt, 2008).

En la actualidad, desde diferentes ámbitos se reconoce el valor de las señales del oyente. A nivel social se ha documentado su valor pragmático porque su desconocimiento ha sido origen de malentendidos comunicativos (Pérez Ruiz, 2011a). En el campo informático se aplican los avances tecnológicos para elaborar diseños informativos, que ayuden a predecir la producción de estas señales (Ward \& Tsukahara, 2000); además, en Japón han sido incorporados en las muestras de habla de los robots (Fujie, 2004). Desde hace tiempo, en el área médica se reconoce su valor 
diagnóstico y terapéutico en la salud mental (Turkstra, Ciccia \& Seaton, 2003; Van Beek, 2006). Por último, en la didáctica de lenguas se empieza a considerar como un factor que puede promover la interacción oral en el aula (Garcés \& Bou, 2004; Sayer, 2005; Mori \& Hayashi, 2006; Wolf, 2008). Sin embargo, a nivel investigador persisten numerosas controversias, que demandan nuevos estudios empíricos para ayudar a clarificar el tema.

\subsection{Turnos de apoyo}

En este apartado se realizará un breve recorrido por los diferentes debates que siguen abiertos sobre las señales verbales del oyente. En la parte final, de manera precisa, se justificará la terminología y clasificación que hemos seguido para la realización de este estudio.

El primer punto de controversia es la diversidad terminológica publicada. Fujimoto (2007) encontró 24 términos diferentes para designar las señales del oyente; y Pérez Ruiz (2011a), en su revisión del tema, halló 40 denominaciones. El término más empleado en las publicaciones en lengua inglesa ha sido backchannel. En japonés existe una mayor uniformidad, ya que suelen denominarse aizuchi (Kita \& Ide, 2007). En español, Vázquez (2000) acuñó el término 'respuestas mínimas reguladoras' y Cestero (2000a) estableció el término 'turnos de apoyo'.

En segundo lugar, se observan problemas derivados sobre su catalogación exacta en la estructura de la conversación. Por un lado, no existe un único criterio para considerarlos como turnos o no (Vázquez, 2000; McCarthy, 2003; Iida, 2005). Por otro lado, sigue habiendo polémica sobre su relación con la superposición de habla (Berry, 1994; Ikeda, 2007). El tercer punto de controversia ha sido cómo definir las funciones de las señales del oyente. Los investigadores no han llegado a un acuerdo sobre las diferentes funciones y clasificaciones del tema (Tao \& Thompson, 1991; Clancy, Thompson, Suzuki \& Tao, 1996; Gardner, 2002; Heinz, 2003; Ishida, 2006; Wong \& Peters, 2007). Pérez Ruiz (2011a), por su parte, documentó 13 funciones diferentes en los trabajos publicados sobre el tema: (1) las señales del oyente pueden actuar como continuadores manteniendo abierto el turno del hablante, (2) expresar la recepción del mensaje del hablante, (3) marcadores, (4) cambio de actividad, (5) valoración, (6) entendimiento, (7) acuerdo, (8) desacuerdo, (9) clarificación, (10) confirmación, (11) interés o atención, (12) final colaborativo, y (13) una respuesta emocional.

A continuación, enumeraremos otros factores sobre los que se discute y se investiga. El primero que citamos es la interculturalidad. Aunque es un fenómeno universal, descrito en todas las lenguas (Levinson, 1983), todavía no se ha clarificado su especificidad lingüística y cultural en distintas lenguas (Oreström, 1983; Clancy et al., 1996; Heinz, 2003; Wolf, 2008). La diferente producción en lengua materna y en L2, así como el fenómeno de la transferencia sociolingüística son temas que siguen 
sin obtener una respuesta uniforme (Heinz, 2003; Krause-Ono, 2004; Iida, 2005; $\mathrm{Li}, 2006)$. Otro factor en donde se han obtenido resultados dispares ha sido el papel del sexo del interlocutor (Cutrone, 2005; Van Beek, 2006; Tanaka, 2007; Pérez Ruiz, 2012). Por último, enumeramos otros tres factores publicados: el tipo de conversación (Ward \& Tsukahara, 2000; Heinz, 2003), la edad (Hess \& Johnson, 1988; Van Beek, 2006) y la influencia del hablante (Cestero, 2000a).

Ante esta compleja situación concluiremos el marco teórico explicando cuáles han sido la terminología y tipología adoptadas en nuestro trabajo. La definición y clasificación empleadas han seguido las indicaciones publicadas por Cestero (Cestero, 2000a, 2000b, 2005). El primer argumento, que justifica nuestra elección, se basa en la rigurosidad de los trabajos empíricos en español realizados por Cestero. Estos estudios muestran la validez de la mencionada clasificación para la lengua española. La segunda razón procede de varios trabajos de investigación en ELE (Inglés, 2010; Pérez Ruiz, 2011a; Pascual, 2012), los cuales han acreditado la idoneidad de la terminología y de la clasificación. Por último, siguiendo esta línea de investigación, en la actualidad se están llevando a cabo nuevos proyectos que están analizando la producción de TA en diversas lenguas y su relación en ELE (Cestero, 2012).

Al tenor de lo indicado, en este artículo denominaremos a las señales del oyente 'turnos de apoyo', concepto acuñado por Cestero (2000a) y que definimos como las vocalizaciones léxicas y no léxicas emitidas por el oyente durante la interacción oral, cuya función primordial es mostrar seguimiento en el acto comunicativo y participación activa en la comunicación (Cestero, 2000a, 2000b). En segundo lugar, la clasificación que hemos seguido en esta investigación ha sido la propuesta por Cestero (2000a), la cual califica a los TA siguiendo tres criterios bien diferenciados: la función de los TA, la complejidad o estructura lingüística de los TA y la influencia del contexto en la producción de los TA. Cada uno de estos criterios será debidamente explicado durante la exposición de los resultados obtenidos.

\section{Metodología}

Nuestro estudio se puede catalogar como una investigación primaria, transversal, básica y descriptiva que ha seguido la metodología empírica e inductiva indicada por el AC y las técnicas procedentes de la Sociolingüística Variacionista (Moreno, 1990; Cestero, 2000a; Griffin, 2005; Hutchby \& Wooffit, 2008). Se considera descriptiva, para diferenciarla de futuras investigaciones experimentales, ya que en esta ocasión no se ha manipulado la variable independiente (nivel lingüístico en la lengua meta) para analizar su impacto sobre la variable dependiente, los TA verbales (Bernardo \& Calderero, 2000).

Los resultados de la investigación están basados en un corpus oral de 30 conversaciones diádicas, mantenidas en español entre estudiantes taiwaneses de ELE. Se han seguido de manera rigurosa cada una de las fases que componen la construcción 
de un corpus oral: (1) selección de una muestra, (2) grabación, (3) transcripción y etiquetado; (4) codificación del fenómeno de estudio, es decir, los TA verbales; y por último, análisis cuantitativo y cualitativo de los resultados obtenidos.

\subsection{Participantes}

La investigación se basa en una muestra no probabilística, de muestreo intencionado (Moreno, 1990) y accidental (Bernardo \& Calderero, 2000). La entidad social de estudio está compuesta por una muestra de 60 estudiantes taiwaneses de español procedente de las cuatro universidades que disponen de Departamento de Español en Taiwán, a saber, la universidad de Furen y Tamkang en Taipéi, la universidad de Chin-Yi en Taichong y la universidad Wenzao en Kaohsiung. La selección de informantes se llevó a cabo siguiendo criterios sociológicos y lingüísticos. Las variables sociológicas que se controlaron para lograr una adecuada validez interna fueron las siguientes: lugar de origen y de residencia, edad, estatus social, motivación para aprender español, nivel educativo, situación de igualdad de poder y solidaridad entre los participantes (Moreno, 1990). Las variables lingüísticas fueron la lengua materna chino mandarín y el nivel de adquisición lingüística en ELE, B1 y B2 del MCER.

Todos los informantes eran hablantes de origen chino, que habían nacido y vivían en Taiwán, nunca habían vivido en un país de lengua española más de dos meses, el rango de edad fue de 20 a 23 años, pertenecían a la clase media taiwanesa y todos eran estudiantes universitarios de español. En este estudio no se tuvo en cuenta el sexo de los interlocutores ${ }^{3 .}$

La muestra fue dividida en dos grupos, denominados A y B, según el nivel lingüístico en ELE. El Grupo A lo componían 30 estudiantes con un nivel B1 en ELE, que participaron en 15 conversaciones diádicas en español. El Grupo B estaba formado por 30 estudiantes de ELE con un nivel de adquisición lingüística B2, que también entablaron 15 conversaciones diádicas en español. La asignación de cada participante a un grupo se determinó siguiendo dos criterios fundamentales. En primer lugar, la certificación de haber aprobado el examen DELE correspondiente. En segundo lugar, en caso de no disponer de los certificados pertinentes, tres profesores de español contribuyeron a la adecuada asignación grupal, siguiendo los criterios de expresión oral propuestos por el MCER (Consejo de Europa, 2002).

\subsection{Selección temática, recolección, transcripción y procesamiento de datos}

Los interlocutores debían comenzar su conversación con un saludo y finalizar con una despedida. La selección de temas en las conversaciones grabadas se dejó a criterio de los informantes. No obstante, los interlocutores tuvieron acceso a una lista temática durante los 10 minutos previos a la grabación. La lista ofrecida fue una modificación de los temas utilizados en los módulos temáticos del Proyecto para el Estudio 
Sociolingüístico del Español de España y América (PRESEEA) ${ }^{4}$. Concretamente, fueron 8 temas: vacaciones, cumpleaños, futuro, internet, música, actividades en un día normal y en los fines de semana. Además, se incluyeron tres temas que permitían a los interlocutores debatir sobre los siguientes asuntos propuestos: el dinero es lo más importante en la vida, profesiones más interesantes y, por último, si es mejor hacer deporte o verlo por televisión.

Se grabaron en audio 30 conversaciones diádicas en español, semidirigidas y mantenidas en un contexto académico, con una duración de 10 minutos cada una. Durante la grabación no se detuvo la grabadora en ningún momento, permitiendo de este modo registrar pausas, silencios, lapsos, titubeos, etc. No se realizaron ensayos previos ni los informantes conocían la naturaleza exacta de la investigación. Cada interlocutor participó solamente en una conversación. Se les invitó a que conversaran del modo más natural posible. Además, para minimizar la influencia de la paradoja del observador, las grabaciones se realizaron en lugares habituales de los interlocutores, los informantes eligieron libremente a su interlocutor y conocían al observador que estaba presente en cada grabación.

Las transcripciones y etiquetado de las grabaciones también han sido realizadas siguiendo las convenciones del grupo PRESEEA. El tiempo de transcripción de cada conversación ha sido de 5 minutos, porque diferentes estudios han mostrado que este periodo permite la reaparición y descripción posterior de los apoyos verbales de manera idónea (Clancy et al., 1996; Cestero, 2000a; Cutrone, 2005). Para el Grupo A se grabaron un total de 150 minutos y 53 segundos. El tiempo total transliterado ha sido de 76 minutos y 50 segundos. Para el Grupo B fueron grabados un total de 158 minutos y 13 segundos. El tiempo total de la transcripción ha sido de 76 minutos y 46 segundos. Siguiendo las indicaciones de algunos investigadores (Turkstra et al., 2003), siempre que fue posible, las transcripciones comenzaron en el segundo minuto de las grabaciones, donde los interlocutores parecen prestar menos atención al entorno artificial y la conversación adquiere más naturalidad. Por último, conscientes de las limitaciones y dificultades de las transcripciones de corpus orales (Liddicoat, 2007; Hutchby \& Wooffitt, 2008), cada transcripción fue revisada por dos doctores en lingüística antes de ser realizado el correspondiente análisis.

Finalmente, se procedió a un análisis cuantitativo y cualitativo de los datos obtenidos. Se realizó un análisis estadístico descriptivo e inferencial, a través de la $T$ de Student, que adoptó en toda la investigación un valor de significación estadística del 0.01 .

\section{Resultados}

Antes de exponer los resultados obtenidos, debemos tener en cuenta dos limitaciones fundamentales en este tipo de estudios. En primer lugar, junto a las variables sociolingüísticas consideradas, deben reconocerse la posible influencia en 
los resultados de otras variables latentes, como pueden ser la especificidad cultural (Heinz, 2003) y las diferencias individuales, psicológicas y conductuales de cada interlocutor (Pérez Ruiz, 2011a). En segundo lugar, podríamos preguntarnos cómo es posible determinar un estándar en los patrones comunicativos en L2 si desconocemos su desempeño habitual en su lengua materna. Esta segunda limitación ha sido tenida en cuenta en el diseño de nuestro corpus oral bilingüe, donde los mismos interlocutores conversaron en ambas lenguas. De esta manera, ha sido posible conocer las estrategias de producción en L1 y en L2.

Dado que excede el cometido de este artículo, simplemente nos limitaremos a mencionar que en las conversaciones en chino del Grupo A y del Grupo B no se obtuvieron diferencias significativas $(\mathrm{p}>0,01)$ para las siguientes variables: número de caracteres chinos emitidos, turnos totales (TT), turnos de habla (TH), superposición de habla $(\mathrm{SH})$, TA verbales y su producción en relación a los lugares de transición pertinente (LTP). Estos datos indican que los estudiantes taiwaneses, con un diferente nivel en ELE, desarrollaron un estilo conversacional similar en la lengua materna. Al final del apartado de resultados se describirá el patrón comunicativo hallado en L1 y su similitud con el obtenido en L2.

\subsection{Aportaciones del oyente a la interacción oral en ELE}

La exposición de los resultados obtenidos se ceñirá a los dos objetivos específicos que han dirigido esta investigación. Por un lado, analizaremos la contribución de las señales lingüísticas y metalingüísticas del oyente a la IO en ELE. Por otro lado, se ofrecerán los datos más relevantes sobre la influencia que el nivel en la L2 ha desempeñado sobre las estrategias comunicativas exhibidas por el oyente. Para llevar a cabo nuestro primer cometido hemos analizado la contribución del oyente a la $\mathrm{IO}$, aportando datos sobre la producción de TA verbales en relación a las siguientes variables: TT, TH, LTP, SH y número de palabras emitidas (PE).

\subsubsection{Turnos totales, turnos de habla, palabras emitidas y turnos de apoyo}

En términos generales, las diferencias cuantitativas y cualitativas halladas entre las conversaciones de ambos grupos han sido notables. La IO del Grupo A ha estado basada en turnos de escasa extensión, muchas veces reducidos a turnos conectados mediante una pregunta y respuesta, con abundantes problemas de interacción, duda y titubeo. Los informantes del Grupo B han emitido turnos más extensos y cualitativamente de mayor complejidad formal, han interactuado mucho más y lo han hecho con bastante más fluidez y naturalidad.

Antes de analizar los TA, ofreceremos datos generales que nos permitirán enmarcar los TA en el contexto de los TT, TH y PE en cada uno de los grupos analizados. En el Grupo A de nuestro corpus, los estudiantes de nivel B1 han producido 724 TT, $518(71,5 \%)$ fueron TH y $206(28,5 \%)$ TA. La media por informante fue de 6.8 TA 
y el rango por informante fue de 2 a 15 TA. En el Grupo B, los estudiantes de nivel B2 durante sus conversaciones en español emitieron un total de $1.327 \mathrm{TT}$, de los cuales, 907 (68\%) fueron TH y 420 (32\%) TA. La media por informante fue de 14 TA y el rango, igualmente por informante, fue de 2 TA a 37 TA. Sobre estos datos hemos realizado un análisis estadístico inferencial de la media, desviación típica y $T$ de Student. La Tabla 1 recoge los valores obtenidos para cuatro variables: la producción de los TT, TH, TA y TA que sirvieron para iniciar un TH. Se han marcado en negrita las variables para las que se obtuvieron una significación estadística del 0.01.

Tabla 1. Grupos A y B. Turnos totales, de habla y de apoyo.

\begin{tabular}{|c|c|c|c|c|}
\hline & Grupo & Media & Desviación Típica & T de Student \\
\hline \multirow{2}{*}{ Turnos totales } & A & 24.13 & 6.786 & \multirow{2}{*}{.000} \\
\hline & B & 44.23 & 14.481 & \\
\hline \multirow{2}{*}{ Turnos de habla } & A & 17.27 & 6.017 & \multirow{2}{*}{.000} \\
\hline & B & 30.23 & 11.159 & \\
\hline \multirow{2}{*}{ Turnos de apoyo } & $\mathrm{A}$ & 6.87 & 3.235 & \multirow{2}{*}{.000} \\
\hline & B & 14.00 & 8.267 & \\
\hline \multirow{2}{*}{ TA inicia $\mathrm{TH}$} & A & 1.47 & 1.106 & \multirow{2}{*}{.265} \\
\hline & B & 1.97 & 2.157 & \\
\hline \multirow{2}{*}{ TA inicia $\mathrm{TH}$ relacionado } & A & 1.27 & 1.015 & \multirow{2}{*}{.637} \\
\hline & B & 1.43 & 1.633 & \\
\hline \multirow{2}{*}{ TA inicia TH cambio de tema } & A & .20 & .407 & \multirow{2}{*}{.101} \\
\hline & $\mathrm{B}$ & .53 & 1.008 & \\
\hline $\mathrm{n}=30 \mathrm{p}<0.01$ & & & & \\
\hline
\end{tabular}

Los resultados indican que las diferencias de medias de los TT, de los TH y de los TA han sido significativas $(\mathrm{p}<0.01)$ entre ambos grupos. Estos resultados muestran que en las conversaciones del Grupo B ha habido más producción de habla. Además, los oyentes del Grupo B han realizado una mayor contribución a la construcción de la interacción oral, registrando más TA verbales que los estudiantes del Grupo A.

Turkstra et al. (2003), en conversaciones en inglés mantenidas entre adolescentes, encontraron un TA verbal cada 5 TH. Nuestros datos muestran la producción de 1 TA cada 3.5 TH en el Grupo A, y una proporción de 1 TA cada 3.2 TH en el Grupo B. Resultados similares han sido obtenidos en una muestra de estudiantes ingleses de ELE, donde se ha documentado 1 TA por cada 3.1 TH (Inglés, 2010); y en un grupo de estudiantes italianos de ELE ha sido publicada la producción de 1 TA por cada 3.5 TH (Pascual, 2012).

La mayor proporción de TA en los estudiantes del Grupo B, que poseen una mayor competencia comunicativa, está en consonancia con lo publicado por Young 
y Lee (2004), para quienes la habilidad de la producción de los TA está en relación con la participación en un mayor número de conversaciones. Por otro lado, también es destacable que en ambos grupos ha habido informantes que solo han emitido 2 TA. Estos datos parecen indicar que factores como la variabilidad individual deben de ejercer alguna influencia en la producción de los TA. Este punto de la variabilidad individual ya fue subrayado por Cutrone (2005) cuando enumeró los siguientes factores que intervienen en la producción de los TA: la cultura, la negociación de la toma de turno, la habilidad lingüística en la lengua meta y la personalidad. Evidentemente, la personalidad de cada informante parece haber influido en la emisión de TA en nuestro corpus. Sin embargo, aunque es un factor al que se le reconoce su presencia, no invalida los resultados obtenidos a través de análisis estadísticos (Heinz, 2003).

También hemos contabilizado el número de PE en cada serie y su relación con los TA. El número total de PE en el grupo A fue de 6.308. En el Grupo B, se contabilizaron un total de 7.978 PE. Se obtuvieron diferencias estadísticamente significativas entre ambos grupos $(\mathrm{p}<0.01)$. Debemos destacar que las conversaciones con mayor número de $\mathrm{PE}$ fueron aquellas en las que se produjeron mayor número de TA. Estos datos obtenidos están en consonancia con los resultados de Wolf (2008), investigador que encontró relación entre la producción de los TA y la mayor producción de PE. Wolf analizó la influencia de los TA en la fluidez oral de tareas orales realizadas por nativos japoneses que aprendían inglés como L2; sus resultados mostraron que los TA contribuían a una mayor producción de habla. Recientemente, Inglés (2010) también ha hallado una relación similar entre la producción de TA y la IO en estudiantes ingleses de ELE.

\subsubsection{Lugares de transición pertinente, superposición de habla y turnos de apoyo}

A continuación, mostraremos los resultados logrados al comparar la producción de TA en los grupos A y B en relación a los LTP y a la SH. La Tabla 2 refleja los datos estadísticos obtenidos. En ambos grupos, los TA se produjeron mayoritariamente en relación con los LTP y sin SH. Concretamente, en el Grupo A, el 81\% de los TA se emitieron en los LTP como alternancias propias y el $76 \%$ de los TA fueron producidos sin SH. En el Grupo B, el 85,5\% de los TA se registraron como alternancias propias en los LTP y el $81 \%$ fueron realizados sin SH. Ambos grupos siguieron un patrón similar de respetar los LTP y de evitar la SH en el momento de emitir los TA. De esta manera, la significación estadística que observamos en la $T$ de Student $(\mathrm{p}<0.01)$ para las variables LTP y emisión de TA sin SH viene motivada por la diferencia numérica en la media de producción de los TA de los dos grupos analizados. También observamos que en el Grupo A, en 13 de las 15 conversaciones analizadas, existió una relación directa entre los TA y la SH. En el Grupo B, también se halló dicha relación en 12 conversaciones. Parece, pues, que en las conversaciones donde hubo mayor interacción oral se produjo mayor superposición de habla y también se emitieron más TA. 
Tabla 2. Grupos A y B. Lugares transición pertinente, superposición y apoyos.

\begin{tabular}{|c|c|c|c|c|}
\hline & Grupo & Media & Desviación Típica & T de Student \\
\hline \multirow{2}{*}{ LTP Apropiado } & A & 5.57 & 2.712 & \multirow{2}{*}{.000} \\
\hline & $\mathrm{B}$ & 11.97 & 7.049 & \\
\hline \multirow{2}{*}{ LTP Inapropiado } & A & 1.30 & 1.489 & \multirow{2}{*}{.102} \\
\hline & B & 2.03 & 1.903 & \\
\hline \multirow{2}{*}{$\begin{array}{c}\text { Inapropiado } \\
\text { Justificado }\end{array}$} & A & 1.10 & 1.322 & \multirow{2}{*}{.133} \\
\hline & $\mathrm{B}$ & 1.70 & 1.705 & \\
\hline \multirow{2}{*}{$\begin{array}{l}\text { Inapropiado } \\
\text { No justificado }\end{array}$} & A & .20 & .484 & \multirow{2}{*}{.399} \\
\hline & B & .33 & .711 & \\
\hline \multirow{2}{*}{ TA sin superposición } & A & 5.2 & 2.413 & \multirow{2}{*}{.000} \\
\hline & B & 11.13 & 6.618 & \\
\hline \multirow{2}{*}{ TA superposición } & A & 1.67 & 1.807 & \multirow{2}{*}{.082} \\
\hline & $\mathrm{B}$ & 2.70 & 2.641 & \\
\hline $\mathrm{n}=30 \mathrm{p}<0.01$ & & & & \\
\hline
\end{tabular}

Nuestros resultados son similares a los publicados por Clancy et al. (1996), quienes encontraron que el 79,5\% de los TA producidos en lengua china respetaban los LTP. Estas cifras fueron muy superiores a las halladas en lengua inglesa (45\%) y japonesa (30,8\%). También Young y Lee (2004) señalaron la preferencia de los coreanos por emitir sus TA en los LTP. Sin embargo, dichos datos difieren de lo publicado por Cestero (2000a), quien contabilizó un 46\% de TA emitidos como alternancias apropiadas en lengua española. La explicación de esta diferencia parece estribar en el diferente estilo comunicativo de los nativos españoles y los estudiantes taiwaneses de español. Así, parece que estos últimos han adoptado un patrón no intrusivo, más típico de su lengua materna (Li, 2006). Por su parte, García (2009) también encontró que las alternancias propias fueron las más frecuentes en las conversaciones en ELE en Alemania, y que los cambios de hablante se produjeron en contextos de conclusión gramatical y sin $\mathrm{SH}$, indicando una búsqueda de armonía por parte de los interlocutores.

Cuando analizamos la emisión de TA en $\mathrm{SH}$, nuestros datos son similares a los hallados por Tao y Thompson (1991) en lengua china; y a los de Rubio (2008), que documentó un 22\% de TA en SH en estudiantes taiwaneses de ELE. Nuestros datos se alejan de los publicados por Oreströn (1983), quien encontró en lengua inglesa un $46,5 \%$ de $\mathrm{TA}$ registrados en $\mathrm{SH}$. También nuestros datos distan de lo publicado en lengua española, donde Berry (1994) halló un 50\% de verbalizaciones realizadas en $\mathrm{SH}$. Una vez más, la justificación procede del modelo comunicativo más reservado de la interacción oral en lengua china.

Los datos obtenidos para los grupos A y B muestran que, independientemente del nivel en la lengua meta, los estudiantes taiwaneses en los grupos A y B mantienen 
un patrón escasamente intrusivo, con enorme respeto por el interlocutor que tiene el turno actual. Los estudiantes de un nivel superior en ELE no han desarrollado las estrategias más intrusivas publicadas por Cestero (2000a) en lengua española. Este hecho, nos hace reflexionar sobre la importancia de introducir la enseñanza explícita de los TA en la clase de ELE, para que los estudiantes sean capaces de desarrollar una estrategia comunicativa más afín con la de la lengua meta.

\subsubsection{Los turnos de apoyo en la comunicación oral}

A modo de breve disertación final de esta primera parte de los resultados, podemos considerar que nuestra investigación ha mostrado que los TA contribuyen a la IO en la lengua meta. Nuestro estudio ha documentado la existencia de una relación entre la producción de TH y de TA. Nuestra elevada proporción de TA con respecto a los TH, y la relación existente con el registro de PE nos permite afirmar, con evidencia empírica, que la conversación no es una sucesión de turnos con contenido referencial, sino que el oyente continuamente realiza una monitorización sobre el enunciado del hablante, tal como defendió Gumperz (1982). De manera que, la IO es el logro compartido del hablante y del oyente, tal como sostuvo Schegloff (1982), dado que ambos contribuyen al flujo comunicativo (Oreström, 1983). Ante esta evidencia teórica y empírica, nuestro trabajo apoya lo publicado por Vázquez (2000), quien defendía que el oyente no es un elemento secundario en la comunicación oral; además, no se puede ofrecer una adecuada comprensión de la IO sin tener en cuenta su contribución, pues su ausencia supondría obtener una visión parcial e insuficiente del acto comunicativo.

La mayor producción de TA en relación con el total de turnos de palabra emitidos, observada en las dos series, indica que el oyente participa en la conversación a través de la recepción del mensaje y de la producción del mensaje. Ambas actividades, de recepción y producción, ya fueron subrayadas por Erickson (1986) y recogidas, posteriormente, por Gallardo (1992), para quien el oyente se convertía en hablante a través de una escucha activa y productiva. De esta manera, y tal y como hemos visto en los dos grupos, el número de los TA que han servido para tomar el TH muestra que el oyente se convierte en un 'oyente combatiente' (Padilla, 2004) que, con una escucha atenta, puede tratar de coger el TH, hecho que también se produce a medida que aumenta el nivel de conocimiento de una segunda lengua.

Para concluir este apartado, debemos subrayar el papel esencial del oyente en la IO. Nuestros resultados han mostrado que, a través de los TA verbales, el oyente realiza una construcción compartida del turno, tal como defendió Gardner (2001), y por lo tanto, son un marcador relevante en la IO.

\subsection{Estrategias comunicativas del oyente en ELE}

En este apartado, se analizará la influencia del nivel de la lengua meta en la producción de los TA verbales. Describiremos, con base empírica y de manera 
cuantitativa y cualitativa, cuáles son las principales estrategias comunicativas del oyente en dos niveles diferentes de competencia comunicativa en ELE. De manera precisa, seguiremos la clasificación adoptada en el marco teórico, que nos permitirá describir las funciones, la estructura lingüística y la influencia del contexto en la emisión de los TA (Cestero, 2000a).

\subsubsection{Funciones de los turnos de apoyo}

En la clasificación de Cestero (2000a) los apoyos conversacionales se distribuían en ocho diferentes funciones: acuerdo, entendimiento, seguimiento, conclusión, recapitulación, conocimiento, reafirmación y apoyos combinados. Los apoyos de acuerdo, como su nombre indica, expresan el acuerdo con el contenido del mensaje en marcha, bien sobre hechos, pensamientos, propósitos, sentimientos, comportamientos y juicios emitidos. La función de los apoyos de entendimiento del oyente es transmitir al hablante la comprensión del enunciado en marcha, sea de una explicación dada, o bien la dentificación de objetos, lugares o hechos mencionados. Los apoyos de seguimiento realizan un seguimiento del mensaje que se está emitiendo y suelen carecer de significado. Mediante los apoyos de conclusión el oyente intenta finalizar el enunciado en marcha o una parte de él, realizando un intercambio cooperativo. A través de los apoyos de recapitulación el oyente resume el enunciado emitido en el turno de habla. En los apoyos de conocimiento el oyente anticipa el contenido que será emitido por el hablante, son posibles gracias al conocimiento general compartido con el interlocutor. Los apoyos de reafirmación suelen ser preguntas formuladas por el oyente (‘¿sí? ¿de verdad?’) cuya finalidad no es asumir el turno de habla, sino solicitar al hablante que confirme y amplíe la información que acaba de emitir. Por último, los apoyos combinados, son emisiones del oyente formadas por dos tipos de apoyos anteriores (Cestero, 2000a, 2005).

En la comparación de funciones realizadas entre los grupos A y B, hemos obtenido que los apoyos de acuerdo fueron los más frecuentes en ambos grupos (el 32,5\% de TA de acuerdo en el Grupo A y el 31,6\% en el Grupo B). Observamos que los dos grupos presentaron diferencias en relación con la segunda y tercera función más frecuentemente hallada. Por un lado, en el Grupo A los TA más frecuentes, después de los TA de acuerdo, fueron los TA de entendimiento (17,5\%), seguidos de los TA de reafirmación $(16 \%)$ y los TA de seguimiento, en cuarto lugar (14\%). Mientras que, para el Grupo B, los TA de seguimiento (28,3\%) ocuparon el segundo lugar en frecuencia, los TA de entendimiento $(13 \%)$ el tercer lugar, y los TA de reafirmación $(7,4 \%)$ fueron los quintos, detrás de los de conclusión (10\%). Por su parte, los TA de conocimiento fueron los menos frecuentes en ambos grupos. En el Gráfico 1 se muestran los valores absolutos y porcentajes obtenidos. 


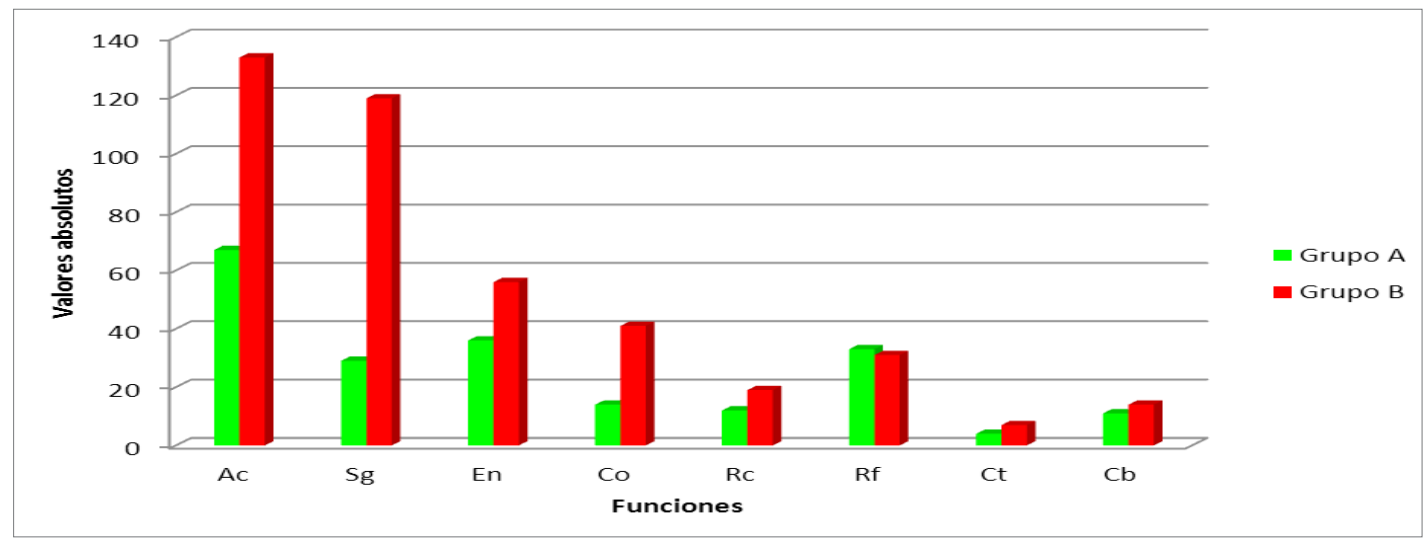

\begin{tabular}{|c|c|c|c|c|c|c|c|c|c|}
\hline & Ac & Sg & En & Co & Rc & Rf & Ct & Cb & Total \\
\hline Grupo A & $67(33 \%)$ & $29(14 \%)$ & $36(17 \%)$ & $14(7 \%)$ & $12(6 \%)$ & $33(16 \%)$ & $4(2 \%)$ & $11(5 \%)$ & 206 \\
\hline Grupo B & $133(31 \%)$ & $119(28 \%)$ & $56(13 \%)$ & $41(10 \%)$ & $19(4 \%)$ & $31(7 \%)$ & $7(2 \%)$ & $14(3 \%)$ & 420 \\
\hline \multicolumn{1}{|c|}{ Ac = Acuerdo. Sg = Seguimiento. En = Entendimiento. Co = Conclusión. } \\
Re = Recapitulación. Rf = Reafirmación. Ct = Conocimiento. Cb = Combinado.
\end{tabular}

Gráfico 1. Valores absolutos y porcentajes de las funciones de turnos de apoyo.

La Tabla 3 muestra los principales hallazgos de la comparación estadística realizada para cada una de las funciones de los TA verbales. Los resultados indican que se han obtenido diferencias estadísticamente significativas entre los grupos A y B en 3 tipos de funciones: $1 .^{\circ}$ los TA de acuerdo $(\mathrm{t}=0.005, \mathrm{p}<0.01) ; 2 .^{\circ}$ los TA de seguimiento $(\mathrm{t}=$ $0.003, \mathrm{p}<0.01) ; \mathrm{y} 3^{\circ}{ }^{\circ}$ los TA de conclusión $(\mathrm{t}=0.006, \mathrm{p}<0.01)$. 
Tabla 3. Grupos A y B. Comparación de las funciones.

\begin{tabular}{|c|c|c|c|c|}
\hline & Grupo & Media & Desviación Típica & T de Student \\
\hline \multirow{2}{*}{ Acuerdo } & A & 2.23 & 1.716 & \multirow{2}{*}{.005} \\
\hline & B & 4.43 & 3.711 & \\
\hline \multirow{2}{*}{ Seguimiento } & A & .97 & 1.098 & \multirow{2}{*}{.003} \\
\hline & B & 3.97 & 4.888 & \\
\hline \multirow{2}{*}{ Entendimiento } & A & 1.20 & 1.215 & \multirow{2}{*}{.140} \\
\hline & B & 1.87 & 2.113 & \\
\hline \multirow{2}{*}{ Conclusión } & A & .47 & .776 & \multirow{2}{*}{.006} \\
\hline & B & 1.37 & 1.520 & \\
\hline \multirow{2}{*}{ Recapitulación } & A & .40 & .675 & \multirow{2}{*}{.294} \\
\hline & B & .63 & .999 & \\
\hline \multirow{2}{*}{ Reafirmación } & A & 1.10 & 1.494 & \multirow{2}{*}{.853} \\
\hline & B & 1.03 & 1.273 & \\
\hline \multirow{2}{*}{ Conocimiento } & A & .13 & .346 & \multirow{2}{*}{.414} \\
\hline & B & .23 & .568 & \\
\hline \multirow{2}{*}{ Combinados } & A & .37 & .669 & \multirow{2}{*}{.536} \\
\hline & B & .47 & .571 & \\
\hline \multirow{2}{*}{ Variedad } & A & 3.57 & 1.455 & \multirow{2}{*}{.019} \\
\hline & B & 4.50 & 1.548 & \\
\hline $\mathrm{n}=30 \mathrm{p}<0.01$ & & & & \\
\hline
\end{tabular}

Varias son las lecturas que podemos hacer de los datos obtenidos. En primer lugar, para ambos grupos los TA son, sobre todo, una estrategia comunicativa por medio de la cual el oyente expresa su acuerdo al enunciado en marcha. Este dato muestra que la definición adoptada en el marco teórico y procedente de Cestero (2000a) es válida para estudiantes de segundas lenguas. En segundo lugar, los datos parecen indicar que el nivel en la lengua meta ha sido un factor relevante en el tipo de función realizado por ambos grupos. Para el Grupo A, su menor nivel en la lengua meta ha determinado que se sumen a la estrategia de acuerdo, las de entendimiento y de reafirmación, pues ambas están en directa relación con la comprensión del enunciado en marcha. Los estudiantes del Grupo A han estado más preocupados por entender y lograr una adecuada reafirmación del enunciado que por mostrar el seguimiento de la conversación o interés por la comunicación en sí. Por el contrario, los estudiantes del Grupo B, que ya tienen un mayor nivel en la lengua meta, al no tener problemas a la hora de comprender ni sentir la necesidad de reafirmar el enunciado en marcha, han desarrollado conversaciones más naturales y espontáneas, con un mayor seguimiento del enunciado en marcha.

Pero, junto a estas estrategias diferentes, también hemos observado diferencias cualitativas, que indican el papel relevante del nivel en la lengua meta para la producción de los TA en ELE. Solamente, a modo de ejemplo, presentamos dos muestras significativas. Los TA de acuerdo del grupo A, en la mayoría de las ocasiones, se limitaron al empleo de adverbios de afirmación (sí) o señales metalingüísticas (mm). 
Mientras que, el Grupo B expresó su acuerdo con un surtido variado de mecanismos verbales y no verbales, tal como recogen los siguientes ejemplos:

(1) Conversación WZ03: Línea 84, 86 y 88

\begin{tabular}{lll}
83 & $15 \mathrm{H}:$ & trabajo \\
\hline 84 & $91 \mathrm{M}:$ & estoy de acuerdo contigo \\
85 & $15 \mathrm{H}:$ & y/ también: el gasto es más:/ alto \\
86 & $91 \mathrm{M}:$ & ah: sí y/ el tiempo: eh: hace fa-fatal en Taipéi también siempre/ \\
87 & & llueve: muchísimo \\
\hline 88 & $15 \mathrm{H}:$ & Sí
\end{tabular}

(2) Conversación WZ012: Línea 66 y 67

\begin{tabular}{lll}
$\mathbf{6 3}$ & $105 \mathrm{M}:$ & ahora es: octubre pero también: hace mucho calor: en Kaohsiung \\
\hline 64 & $106 \mathrm{M}:$ & sí/casi siempre:/ (m:) \\
65 & $105 \mathrm{M}:$ & en Kaohsiung no: hay: \\
66 & $106 \mathrm{M}:$ & no hay invierno \\
\hline 67 & $105 \mathrm{M}:$ & no/ seguro/ seguro
\end{tabular}

Estos ejemplos del Grupo B muestran la construcción compartida de la conversación por el hablante y el oyente. En el ejemplo (1), el oyente no se limita a utilizar un adverbio de afirmación, sino que construye una secuencia con dos tipos diferentes de acuerdos. Comienza con una frase que muestra un mayor nivel en ELE: 'estoy de acuerdo contigo'. Después, expresa su acuerdo: 'ah, sí' y toma el TH. En el segundo ejemplo, ambos interlocutores han expresado en su acuerdo un mayor nivel en la lengua meta, primero el informante 106M: 'sí, casi siempre’. Y en las líneas 65 y 67, respectivamente, el otro informante ha realizado un TA de conocimiento seguido de un acuerdo sobre un enunciado negativo: 'no, seguro, seguro'. En este sentido, debemos subrayar la dificultad que supone expresar un acuerdo sobre un enunciado negativo.

\subsubsection{Estructura lingüística de los turnos de apoyo}

El segundo de los criterios de la clasificación de Cestero (2000a) divide los TA según su forma lingüística en apoyos simples, complejos y compuestos. Los TA simples son expresados mediante un elemento paralingüístico o una palabra. Los apoyos complejos están constituidos por una secuencia de dos o más palabras, con una única unidad sintáctica y semántica. Los TA compuestos están formados por más de una unidad sintáctica y semántica.

Al analizar la estructura lingüística de los TA observamos que, en los grupos A y $\mathrm{B}$, los TA verbales emitidos han sido expresados en su mayoría como apoyos simples. En el Grupo A, fueron 130 apoyos simples (el 63\%), mientras que en el Grupo B fueron 288 TA simples, es decir, el 68,5\% del total de TA. En segundo lugar, se 
produjeron los apoyos complejos, 38 casos (el 18,5\%) en el Grupo A y 88 casos (el $21 \%$ ) en el Grupo B. En tercer lugar, encontramos los apoyos compuestos, de los que, en el Grupo A se contabilizaron los mismos casos que de apoyos complejos; mientras que en el Grupo B fueron 44 casos (el 10,5\%), es decir, la mitad de los apoyos complejos. En la Tabla 4 aparecen los datos obtenidos.

Tabla 4. Grupos A y B. Complejidad lingüística de los turnos de apoyo.

\begin{tabular}{|c|c|c|c|c|}
\hline & Grupo & Media & Desviación Típica & T de Student \\
\hline \multirow{2}{*}{ Simples } & A & 4.33 & 2.057 & \multirow{2}{*}{.000} \\
\cline { 2 - 5 } & B & 9.60 & 6.196 & \\
\hline \multirow{2}{*}{ Complejos } & A & 1.27 & 1.143 & \multirow{2}{*}{.012} \\
\cline { 2 - 5 } & B & 2.93 & 3.248 & \multirow{2}{*}{.544} \\
\hline \multirow{2}{*}{ Compuestos } & A & 1.27 & 1.202 & \multirow{2}{*}{} \\
\cline { 2 - 5 } & B & 1.47 & 1.332 & \\
\hline $\mathrm{n}=30 \mathrm{p}<0.01$. & & & & \\
\hline
\end{tabular}

Los datos muestran que, para la $T$ de Student, se obtuvieron diferencias estadísticas en la diferencia de las medias de los apoyos simples $(\mathrm{t}=000, \mathrm{p}<0.01)$. Para la variable TA complejos, se obtuvo una diferencia estadísticamente significativa al 5\% ( $\mathrm{t}=$ 012, $\mathrm{p}<0.05)$. Para los TA compuestos no se halló significación estadística. Los adverbios de afirmación y las verbalizaciones no léxicas fueron los apoyos simples más frecuentes en ambas series. En el Grupo A, emitieron el adverbio de afirmación 'sí' en 53 casos (40,7\%), y verbalizaciones no léxicas en el 31\% de los casos, destacando la verbalización 'mm' (16\%). En el Grupo B, produjeron el adverbio de afirmación 'sí' en 83 casos $(29 \%)$, y le siguió, en frecuencia de aparición, el elemento paralingüístico 'mm' (54 casos, 19\%).

Nuestros datos, en relación a la complejidad lingüística, confirman lo publicado sobre la estructura lingüística simple de la mayoría de los apoyos verbales y la verbalización no léxica 'mm' (Oreström, 1983; Clancy et al., 1996; Cutrone, 2005; Wong \& Peters, 2007). Por lo tanto, los apoyos del oyente suelen ser señales simples no interruptivas, que se emiten con el fin de permitir el transcurso fluido y natural de la conversación.

\subsubsection{Influencia del contexto en los turnos de apoyo}

La clasificación de los TA de Cestero (2000a) se completa con la influencia del contexto en la producción de los TA verbales. En función de los requerimientos pragmáticos, que intervienen en la producción de los TA, se distinguían TA voluntarios y TA requeridos. Los primeros son emitidos libremente por el oyente; mientras que, en los últimos, su producción viene determinada por la emisión realizada por el hablante. A su vez, estos últimos pueden ser simplemente requeridos, exigidos por la interacción y obligados por la interacción. 
En el Grupo A, solamente $41 \mathrm{TA}$, el 20\%, se realizaron de modo voluntario por el oyente. 109 TA (el 53\%) fueron requeridos, 23 TA (el 11\%) resultaron exigidos pragmáticamente y $33 \mathrm{TA}$ (el 16\%) se emitieron obligados por la interacción. Nuestros resultados en el Grupo B muestran que 271 TA (el 65\%) fueron, de algún modo, requeridos por la IO, mientras que, en 149 ocasiones (el 35\%) el oyente emitió sus TA de manera voluntaria. Cuando desglosamos los TA requeridos, hallamos que la mayoría fueron requeridos pragmáticamente, en concreto, 200 casos (el 47,7\%). Por otro lado, fueron exigidos pragmáticamente en 39 casos (el 9\%) y obligados por la interacción en 32 casos (el 7,7\%). A continuación, en la Tabla 5 ofrecemos los datos más significativos relativos a la comparación estadística llevada a cabo:

Tabla 5. Grupos A y B. Influencia del contexto.

\begin{tabular}{|c|c|c|c|c|}
\hline & Grupo & Media & Desviación Típica & Tde Student \\
\hline \multirow{2}{*}{ Voluntarios } & A & 1.37 & 1.159 & \multirow{2}{*}{.000} \\
\hline & B & 4.97 & 4.484 & \\
\hline \multirow{2}{*}{ Requeridos } & A & 3.63 & 2.109 & \multirow{2}{*}{.001} \\
\hline & B & 6.67 & 4.130 & \\
\hline \multirow{2}{*}{ Exigidos } & A & .77 & .679 & \multirow{2}{*}{.112} \\
\hline & B & 1.30 & 1.664 & \\
\hline \multirow{2}{*}{ Obligados } & A & 1.07 & 1.015 & \multirow{2}{*}{1.000} \\
\hline & $\mathrm{B}$ & 1.07 & 1.337 & \\
\hline $\mathrm{n}=30 \mathrm{p}<0.01$. & & & & \\
\hline
\end{tabular}

Estos datos muestran que hemos obtenido diferencias estadísticamente significativas en la producción de los dos tipos de apoyos más frecuentes de las dos series, esto es, en los apoyos voluntarios $(t=0.000, \mathrm{p}<0.01)$ y los apoyos requeridos pragmáticamente $(\mathrm{t}=0.001, \mathrm{p}<0.01)$. El menor número registrado en las dos series para los otros dos tipos impide obtener una significación estadística.

A tenor de estos resultados, podemos resaltar que, en ambos grupos, los apoyos requeridos pragmáticamente han sido los más frecuentes; de hecho, la mitad de todos los TA de cada serie han sido de este tipo. Este dato confirma que la emisión de los TA no debe ser considerada como una producción voluntaria o marginal al acto comunicativo, sino que viene requerida por la propia interacción oral (Vázquez, 2000). En segundo lugar, las mayores diferencias obtenidas han sido encontradas en la producción de TA obligadas por la interacción. El menor nivel en el Grupo A ha originado numerosos momentos de titubeo, duda e incertidumbre en la consecución del turno. El mayor nivel en el Grupo B parece haber sido un factor determinante para la menor producción de TA obligados pragmáticamente. 


\subsection{Apoyos conversacionales: Nivel lingüístico versus transferencia sociolingüística}

En este apartado presentaremos unas reflexiones finales sobre la génesis y significación de los resultados obtenidos. Por un lado, nuestros datos apoyan lo publicado sobre la relación que existe entre el nivel de adquisición de una segunda lengua y los TA producidos (Clancy et al., 1996; Heinz, 2003; Li, 2006). Igualmente, se ha publicado que el uso de los TA es una de las habilidades que se adquieren más tardíamente (Hess \& Johnston, 1988; Van Beek, 2006). Entre las razones que explicarían esta tardía adquisición y la influencia del nivel lingüístico en la lengua meta se pueden aludir razones neurolingüísticas, como el mayor comportamiento automático que realiza el oyente experimentado en una lengua durante la conversación (Pérez Ruiz, 2011b). Se piensa que esta habilidad se va desarrollando en los interlocutores nativos gracias, entre otros factores, a la participación en un gran número de conversaciones. La participación en un mayor número de conversaciones en L2 también facilita en el oyente la proyección de los LTP (Young \& Lee, 2004); lo cual determina que los alumnos de mayor nivel, gracias a una mayor participación en conversaciones en L2, hayan adquirido mejor la proyección de los LTP y, por lo tanto, sean capaces de producir un mayor número de TA.

Pero estas razones neurolingüísticas sugeridas, por sí solas, no explicarían plenamente las diferencias observadas. Debemos preguntarnos, entonces, además de la influencia del nivel en ELE, ¿de dónde procede la mayor pericia observada en las estrategias del grupo B? Obviamente, no proceden de un conocimiento explícito adquirido, ya que los informantes no habían recibido en clase una instrucción explícita de las estrategias de TA utilizadas por los nativos españoles. De hecho, nuestras emisiones registradas difirieron de los patrones utilizados por los nativos españoles (Cestero, 2000a). Entonces, sería plausible sugerir que adoptaron estrategias procedentes de su lengua materna. Este aspecto fue estudiado por Pérez Ruiz (2011b), quien comparó las mismas 15 conversaciones en español del Grupo B con 15 conversaciones mantenidas en su lengua materna, chino mandarín, por los mismos interlocutores del Grupo B, y que se denominó Grupo C. Aquel análisis comparativo, entre las conversaciones en español y chino mantenidas por los mismos interlocutores, mostró, como principal conclusión, que en las conversaciones en español los estudiantes del Grupo B transfirieron estrategias comunicativas de apoyos verbales procedentes de su lengua materna, tal como lo muestra la correlación significativa hallada en la producción de TA, de los LTP apropiado, TA sin superposición y patrones similares de TA de acuerdo y seguimiento en ambas lenguas. Los estudiantes adoptaron el estilo comunicativo menos intrusivo que caracteriza la $\mathrm{IO}$ en lengua china (Clancy et al., 1996; Li, 2006). En la Tabla 6 se muestran algunos de los datos más relevantes obtenidos. 
Tabla 6. Grupos B (conversaciones en español) y C (conversaciones en chino).

\begin{tabular}{|c|c|c|c|c|c|c|}
\hline & Grupo & Media & $\begin{array}{l}\text { Desviación } \\
\text { Típica }\end{array}$ & $\begin{array}{c}\text { Correlación } \\
\text { Pearson }\end{array}$ & $\begin{array}{l}\text { Significación } \\
\text { Pearson }\end{array}$ & $T$ de Student \\
\hline \multirow{2}{*}{ Turnos de apoyo } & B & 14.00 & 8.27 & \multirow{2}{*}{0.589} & \multirow{2}{*}{0.001} & \multirow{2}{*}{0.014} \\
\hline & $\mathrm{C}$ & 18.27 & 10.82 & & & \\
\hline \multirow{2}{*}{ LTP Apropiado } & B & 11.97 & 7.05 & \multirow{2}{*}{0.600} & \multirow{2}{*}{0.000} & \multirow{2}{*}{0.002} \\
\hline & $\mathrm{C}$ & 16.73 & 9.46 & & & \\
\hline \multirow{2}{*}{$\begin{array}{c}\text { TA sin } \\
\text { superposición }\end{array}$} & B & 11.30 & 6.61 & \multirow{2}{*}{0.548} & \multirow{2}{*}{0.002} & \multirow{2}{*}{0.009} \\
\hline & $\mathrm{C}$ & 14.80 & 7.62 & & & \\
\hline \multirow{2}{*}{ Acuerdo } & B & 4.43 & 3.71 & \multirow{2}{*}{0.710} & \multirow{2}{*}{0.000} & \multirow{2}{*}{0.000} \\
\hline & $\mathrm{C}$ & 9.07 & 5.89 & & & \\
\hline Seguimiento & $\begin{array}{l}\mathrm{B} \\
\mathrm{C}\end{array}$ & $\begin{array}{l}3.97 \\
3.87\end{array}$ & $\begin{array}{l}4.89 \\
5.12\end{array}$ & 0.504 & 0.004 & 0.913 \\
\hline $\mathrm{n}=30$ & & & & & & \\
\hline
\end{tabular}

Estos datos nos permiten considerar que, junto al nivel en la lengua meta y el mayor automatismo neurológico en las emisiones de los TA, debe de ser también tenido en cuenta el fenómeno de la transferencia sociolingüística en la génesis de las estrategias de producción de las señales del oyente. El peso específico de cada factor exigirá nuevos estudios para analizar estos y otros aspectos involucrados en el origen de las respuestas verbales del oyente.

En segundo lugar, la riqueza sociolingüística y cultural del español exige cautela en relación a las mencionadas estrategias más intrusivas halladas por Cestero (2000a), ya que sus resultados corresponden a hablantes nativos de España. Estudios posteriores deberán dilucidar si estas estrategias de mayor interrupción y colaboración en la construcción del turno de habla, obtenidas por Cestero, son inherentes al español o se trata de un rasgo sociocultural de una región determinada o de un determinado tipo de hablante.

Por último, los resultados obtenidos en nuestra investigación justificarían la incorporación didáctica de los apoyos verbales propios de la lengua española en las clases de conversación de ELE, para que los estudiantes tomen conciencia del fenómeno, de su transferencia y de la especificidad sociolingüística en cada lengua.

\section{CONCLUSIONES}

En este artículo se ha analizado el significado de uno de los factores menos estudiado en la adquisición de la competencia comunicativa en español como lengua extranjera, a saber, la contribución de las señales verbales del oyente a la comunicación oral en ELE. De manera rigurosa, a través de un corpus oral de 30 conversaciones diádicas, mantenidas por estudiantes taiwaneses de dos niveles lingüísticos diferentes en ELE, se han ofrecido datos sobre el papel que la escucha atenta y participativa realizada por los oyentes ejerce sobre la interacción oral en ELE. 
Al tenor de los datos obtenidos, podemos afirmar que los estudiantes con mayor competencia comunicativa muestran mayor producción lingüística, a la vez que, realizan una escucha más atenta y participativa, lo que contribuye a la mayor espontaneidad y naturalidad en las conversaciones. Los estudiantes taiwaneses de mayor nivel en ELE producen conversaciones con mayor grado de IO, mayor número de apoyos verbales y mayor interés por el hecho conversacional en sí mismo; desarrollando estrategias más diversas y mejor construidas de TA para seguir de manera atenta el enunciado del hablante. Por el contrario, los estudiantes de menor nivel en ELE han producido conversaciones menos naturales, y han empleado mecanismos que han estado más relacionados con la comprensión de los enunciados. Estos datos constatan que la habilidad lingüística es un factor determinante en el tipo de producción de los TA verbales. Además, confirman que la adquisición de los TA verbales se produce en estadios más tardíos del aprendizaje. Por último, en el origen del fenómeno cabe aducir razones de mayor automatismo neurológico y la posible existencia de una transferencia sociolingüística.

La evidencia empírica proporcionada en este artículo, sobre la contribución de los TA a la interacción oral en ELE, confirma que la conversación es una construcción compartida en la que ambos interlocutores, hablante y oyente, son esenciales al acto comunicativo. Este reconocimiento del valor de las aportaciones del oyente en L2 justificaría abrir una nueva línea de investigación en la que se planteen propuestas didácticas, que puedan incorporar estas señales verbales del oyente en los planes curriculares de la enseñanza de ELE. 


\section{REFERENCIAS BIBLIOGRÁFICAS}

Bangerter, A. \& Clark, H. H. (2003). Navigating joint projects with dialogue. Cognitive Science, 27(2), 195-225.

Bernardo, J. \& Calderero, J. F. (2000). Aprendo a Investigar en Educación. Madrid: Rialp.

Berry, A. (1994). Spanish and American Turn-Taking Styles: A Comparative Study. Pragmatics and Language Learning. Monograph series, 5, 180-190.

Cestero, A. M. (2000a). Los turnos de apoyo conversacionales. Cádiz: Universidad de Cádiz.

Cestero, A. M. (2000b). El intercambio de turnos de habla en la conversación. Análisis sociolingüístico. Alcalá de Henares: Servicio de Publicaciones de la Universidad de Alcalá.

Cestero, A. M. (2005). La conversación y la enseñanza de lenguas extranjeras. Madrid: Arco/ Libros.

Cestero, A. M. (2012). La enseñanza de la conversación en ELE: Estado de la cuestión y perspectivas de futuro. Revista Internacional de Lenguas Extranjeras, 1, 31-62.

Clancy, P. M., Thompson, S. A., Suzuki, R. \& Tao, H. (1996). The conversational use of reactive tokens in English, Japanese, and Mandarin. Journal of Pragmatics, 26(3), 355-387.

Consejo de Europa, (2002). Marco Común Europeo de referencia para las lenguas: Aprendizaje, enseñana, evaluación. Madrid: Secretaría General Técnica del MECDSubdirección General de Información y Publicaciones y Grupo ANAYA, S.A.

Cutrone, P. (2005). A case study examining backchannels in conversations between Japanese-British dyads. Multilingua: Journal of Cross-Cultural and Interlanguage Communication, 24(3), 237-274.

Erickson, F. (1986). Listening and speaking. En D. Tannen \& J. Alatis (Eds.), Georgetown University Round Table on Language and Linguistics (pp. 294-319). Washington DC: Georgetown University Press.

Fujie, S. (2004). A conversation Robot with Bach-channel Feedback Function based on Linguistic and Nonlinguistic Information. Ponencia presentada en el 2nd International Conference on Autonomous Robots and Agents. Palmerston North, Nueva Zelanda.

Fujimoto, D. T. (2007). Listener responses in Interaction: A case of abandoning the term backchannel. Journal of Osaka Jogakuin 2year College, 37, 35-54. 
Gallardo Paúls, B. (1991). Pragmática y análisis conversacional: Hacia una pragmática del receptor. Sintagma, 3, 25-38.

Gallardo Paúls, B. (1992). El dinamismo conversacional: Subsunción y feed-back. Carácter acumulativo de los elementos conversacionales. Comunicación y sociedad, 5(1/2), 51-75.

Garcés, P. \& Bou, P. (2004). A pragmatic account of lListenership: Implications for Foreign/Second Language teaching. Revista alicantina de estudios ingleses, 17, 6-57.

García, M. (2009). La competencia conversacional de estudiantes de español como lengua extranjera. Análisis y propuesta didáctica. Tesis doctoral, Universidad de Alcalá, Madrid.

Gardner, R. (2001). When listeners talk. Amsterdam: John Benjamins.

Gardner, R. (2002). When listeners talk: Response tokens and listener stance. Amsterdam, Netherlands: John Benjamins.

Griffin, K. (2005). Lingüistica aplicada a la enseñanza del español como 2/L. Madrid: Arco/ Libros.

Gumperz, J. J. (1982). Discourse strategies. Cambridge: Cambridge University Press.

Heinz, B. (2003). Backchannel responses as strategic responses in bilingual speakers' conversations. Journal of Pragmatics, 35(7), 1113-1142.

Hess, L. J. \& Johnston, J. R. (1988). Acquisition of back channel listener responses to adequate messages. Discourse Processes: A Multidisciplinary Journal, 11(3), 319-335.

Hutchby, I. \& Wooffitt, R. (2008): Conversation analysis. Cambridge: Polity Press.

Iida, S. (2005). Overlapping in Japanese conversation. Tesis doctoral, University of New South Gales, Gales.

Ikeda, K. (2004). Listenership in Japanese: An examination of overlapping listener response [en línea]. Disponible en: http://nflrc.hawaii.edu/Networks/NW32.pdf

Inglés, B. (2010). El funcionamiento de los turnos de apoyo en la conversación en español de estudiantes ingleses de ELE. Tesis de magíster, Universidad Antonio de Nebrija, Madrid.

Ishida, H. (2006). Learners' perception and interpretation of contextualization cues in spontaneous Japanese conversation: Back-channel cue Uun. Journal of Pragmatics, 38(11), 1943-1981. 
Kita, S. \& Ide, S. (2007). Nodding, aizuchi, and final particles in Japanese conversation: How conversation reflects the ideology of communication and social relationships. Journal of Pragmatics, 39(7), 1242-1254.

Krause-Ono, M. (2004). Change in backchanneling behaviour. The influence from L2 to L1 on the use of backchannel cues. Journal of Cognitive Science. Muroran Cognitive Science Circle, 3, 51-81.

Levinson, S. C. (1983). Pragmatics. Cambridge: Cambridge University Press.

Li, H. Z. (2006). Backchannel responses as misleading feedback in intercultural discourse. Journal of Intercultural Communication Research, 35(2), 99-116.

Liddicoat, A.J. (2007). An introduction to conversation analysis. Londres: Continuum.

McCarthy, M. (2003). Talking back: Small interactional response tokens in everyday conversation. Research on Language and Social Interaction, 36(1), 33-63.

Moreno, F. (1990). Metodología sociolingüística. Madrid: Gredos.

Moreno, F. (1998). Principios de sociolingüistica y sociología del lenguaje. Barcelona: Ariel.

Mori, J. \& Hayashi, M. (2006). The achievement of intersubjectivity through embodied completions: A study of interactions between First and Second Language Speakers. Applied Linguistics, 27(2), 195-219.

Oreström, B. (1983). Turn-talking in English conversation. Lund: LiberFörlang Lund.

Padilla, X. (2004). Del oyente receptor al oyente combatiente. ELUA, 18, 213-230.

Pascual, C. (2012). La producción de turnos de apoyo verbales en la conversación de estudiantes italianos de ELE. Estudio preliminar. Revista Nebrija de Lingüistica Aplicada, 11(6), 144-173.

Pérez Ruiz, J. (2011a). El papel del oyente en la conversación china y española. Un estudio contrastivo sociolingüistico. Taipéi: Central Book.

Pérez Ruiz,J. (2011b). Los apoyos conversacionales: Una aproximación neurolingüística para la clase de español como lengua extranjera. Linred, 9, 1-22.

Pérez Ruiz, J. (2012). Gender differences and listener responses in Spanish foreign language conversations. Languages, Literary Studies and International Studies: An International Journal, 9, 1-24.

Rubio, M. (2008). Interrupción y superposición de habla en estudiantes taiwaneses de ELE. Tesis de magíster, Universidad Antonio de Nebrija, Madrid.

Sayer, P. (2005). An intensive approach to building conversation skills. English Language Teacher Journal, 59(1), 14-22. 
Schegloff, E. (1982). Discourse as an interactional achievement: Somes uses of 'uh, huh' and other things that comes between sentences. En D. Tannen (Ed.), Analizing Discourse Text and Talk (pp. 71-93). Washington, DC: Georgetown University Press.

Seedhouse, P. (2004). The interactional architecture of the language classroom: A conversation analysis perspective. Malden, MA: Blackwell.

Tanaka, K. (2007). Diferential use of reactive tokens in Japanese in turn management and by gender. Japan: Temple University.

Tao, H. \& Thompson, S. A. (1991). English backchannels in Mandarin conversations: A case study of superstratum pragmatic 'interference'. Journal of Pragmatics, 16(3), 209-223.

Turkstra, L., Ciccia, A. \& Seaton, C. (2003). Interactive behaviors in adolescent conversation dyads. Language, Speech and Hearing Services in Schools, 34(2), 117142.

Van Beek, Y. (2006). Gender-specific development of nonverbal behaviours and mild depression in adolescence. Journal of Child Psychology and Psychiatry, 47(12), 1272-1283.

Vázquez, N. (2000). Respuestas mínimas reguladoras: Los límites de la marginalidad. Oralia: Análisis del Discurso Oral, 3, 221-242.

Ward, N. \& Tsukahara, W. (2000) Prosodic features which cue Back-Channel responses in English and Japanese. Journal of Pragmatics: An Interdisciplinary Journal of Language Studies, 32(8), 1177-1207.

Wolf, J. P. (2008). The effects of backchannels on fluency in L2 oral task production. System, 36(2), 279-294.

Wong, D. \& Peters, P. (2007). A study of backchannels in regional varieties of English, using corpus mark-up as the means of identification. International Journal of Corpus Linguistics, 12(4), 479-509.

Young, R. F. \& Lee, J. (2004). Identifying units in interaction: Reactive tokens in Korean and English conversations. Journal of Sociolinguistics, 8(3), 380-407. 


\section{NOTAS}

1 Esta investigación se ha llevado a cabo gracias al proyecto denominado "Analysis of listener verbal responses in bilingual corpora oral. Contrastive study Chinese and Spanish". Proyecto NSC 99-2410-H-160017 del Consejo Nacional de la Ciencia (National Science Council) de Taiwán.

2 El interés por los hallazgos obtenidos en este estudio descriptivo, ha favorecido que el Consejo Nacional de la Ciencia de Taiwán haya aprobado la realización de un estudio experimental, que está analizando una propuesta didáctica de incorporación de los apoyos verbales a la clase de ELE. En concreto, se trata del Proyecto NSC 101-2410-H-160-009- denominado "Learning the listener responses in Spanish classroom and its influence on oral interaction in the mother tongue and Spanish foreign language".

3 Para conocer la influencia del sexo de los interlocutores en la producción de turnos de apoyos se remite al estudio previamente publicado por Pérez Ruiz (2012).

4 Para una mayor profundización sobre la metodología, se puede consultar la página electrónica del Proyecto para el Estudio Sociolingüístico del Español de España y América (PRESEEA) disponible en: http://preseea.linguas.net/. 